\title{
Vorwort zum ersten Teil
}

In dem folgenden Teil des vorliegenden Buches sind die ersten vier Kapitel der Interpretation des naturphilosophischen Hauptwerkes von Plessner gewidmet. In den Stufen des Organischen und der Mensch werden die lebensweltlichen Ermöglichungsstrukturen für bio-anthropologische Vergleiche zwischen der personalen Lebenssphäre von Menschen und non-humanen Lebensformen in der Pflanzen- und Tierwelt rekonstruiert. Diese Naturphilosophie stellt eine phänomenologische und dialektische Hermeneutik der belebten Natur im Unterschied zu und Zusammenhang mit der unbelebten Natur dar. Die Hypothese, dass lebende Körper ihre eigene Grenze realisieren, eröffnet Spektren des Grenzüberganges zwischen vollständiger Abschließung und vollständiger Öffnung des Organismus gegenüber der Umwelt. Sie wird durch verschiedene Organisations- und Positionalitätsformen prozessphilosophisch entfaltet, bis am Ende die exzentrische Positionalitätsform als wirkliche Ermöglichung personalen Lebens erschlossen werden kann, in dem die bio-anthropologischen Vergleiche angestellt werden. Die exzentrische Positionalität besteht funktional gesehen aus einem Strukturbruch, der die Betroffenen in Verschränkungsaufgaben stellt, deren stets erneute Lösung eines geschichtlichen Prozesses bedarf. Diese geschichtliche Verwirklichung der exzentrischen Positionalität kann in den drei anthropologischen Grundgesetzen der natürlichen Künstlichkeit, der vermittelten Unmittelbarkeit und des utopischen Standortes eingesehen und verstanden werden. Die Grenzen der personalen Verhaltensbildung treten in den qualitativen Lebenserfahrungen des ungespielten Lachens und Weinen hervor.

Die Kapitel 5 bis 7 stellen das naturphilosophische Hauptwerk in seinen transdisziplinären Kontext mit Erfahrungswissenschaften, deren Voraussetzungen, Bedingungen und Folgen die moderne personale Lebenssphäre beeinflussen. Es war als ein philosophisch-anthropologischer Beitrag zu der transdisziplinären Rahmenwissenschaft von der Personalität des Menschen konzipiert, die von den Bio- und Medizinwissenschaften über die Sozial- und Kulturwissenschaften bis zu den Geisteswissenschaften hätte reichen können, wäre diese Kooperation dann nicht durch den Nationalsozialismus verhindert worden. Besonderes Augenmerk wird auf die Rekonstruktion der Systematik gelegt, der gemäß sich Plessners Philosophische Anthropologie zur klassischen Psychoanalyse positioniert hat, vor allem kritisch, was den kausalen Erklärungsanspruch, und kooperativ, was den Verstehensanspruch der Psychoanalyse betrifft, woraus sich für die nachklassische Konstellation lernen lässt. Auch Plessners systematische Auseinandersetzungen mit Uexkülls neuer, antidarwi- 
nistischer Biologie geben Gelegenheit, noch immer aktuelle Erweiterungen der Evolutionstheorie in Erinnerung zu rufen. Dies betrifft auch den wichtigen Unterschied und Zusammenhang zwischen biotischen Umwelten, soziokulturellen Umwelten und philosophisch erschlossenen Welten, deren Offenheit fragmentarisch bleibt.

Schließlich komme ich in den Kapiteln 8 bis 10 auf meinen Entwurf der Philosophischen Anthropologie als des lebensweltlichen Forschungsrahmens für die neurobiologische Hirnforschung, die vergleichende Primatologie und die Kognitionswissenschaften der Gegenwart zurück (Krüger 1993, 2007 u. 2010). Nachdem sich der Rauch der Fehlalternative, entweder unbedingte Freiheit oder unbedingter Determinismus, über der öffentlichen Diskussion dieser Hirnforschung von vor eineinhalb Jahrzehnten gelegt hat, kann man zu den ungelösten grundlagentheoretischen Problemen zurückkehren. Die Neurobiologen konnten von Anfang an nicht ihre kausalen Erklärungsansprüche einlösen, da sie zunächst einmal zurecht Modelle entwerfen, nach denen sie die selbstreferentielle Funktionsweise des Gehirnes verstehen können. In dieser Funktionsweise kann das neurophysische Korrelat für diejenige Selbstreferenz bestehen, die auch im personalen Selbstbewusstsein und der Sprache Fremdreferenz ermöglicht. Der Unterschied und Zusammenhang zwischen Mitmachen, Nachmachen und Nachahmen oder Darstellen könnte eine Brücke in die Primatologie bauen, in der die Gemeinsamkeiten und Unterschiede zwischen Menschen und Menschenaffen umstritten bleiben. Schließlich haben sich die Kognitionswissenschaften der beiden letzten Dekaden darum bemüht, ihre Reduktion des Geistes auf das Rechnen dadurch zu relativieren, dass sie die Kognition als embodied, embedded, extended und enactive verstehen. Dabei handelt es sich aus der Philosophischen Anthropologie um willkommene und endliche Wiederentdeckungen.

Wenngleich ich mich im 1. Kapitel der Biophilosophin Marjorie Grene in ihrem Urteil anschließe, dass Plessners Naturphilosophie in ihrer Bedeutung mit den großen Würfen in Alfred North Whiteheads Prozessphilosophie und Maurice Merleau-Pontys Leibesphänomenologie vergleichbar ist, liegt umso mehr eine Parallelentwicklung dieser Philosophischen Anthropologie der Negativität des Absoluten mit John Deweys Metaphysik der Differenz vor. Darauf gehe ich im vierten Teil des vorliegenden Buchs ein. 